\title{
Silicon Photonic Chip for Dynamic Wavelength Division Multiplexed FBG Sensors Interrogation
}

\author{
Y. Marin ${ }^{1, *}$, A. Celik' ${ }^{1}$, S. Faralli ${ }^{1}$, L. Adelmini ${ }^{2}$, C. Kopp ${ }^{2}$, F. Di Pasquale ${ }^{1}$, C. J. Oton ${ }^{1}$ \\ ${ }^{1}$ Scuola Superiore Sant'Anna, TECIP Institute, Via G. Moruzzi 1, 56124 Pisa, Italy \\ ${ }^{2}$ CEA LETI, Minatec, 17 rue des Martyrs, Grenoble, 38054 France \\ Author e-mail address: * y.marin@santannapisa.it
}

\begin{abstract}
We demonstrate wavelength division multiplexed Fiber Bragg Gratings interrogation using an integrated unbalanced Mach-Zehnder Interferometer and an arrayed waveguide grating on silicon-on-insulator, showing a good performance agreement when compared to a commercial readout unit.

OCIS codes: (130.6010) Sensors; (120.5060) Phase modulation; (060.3735) Fiber Bragg gratings.
\end{abstract}

\section{Introduction}

Fiber Bragg grating (FBG) sensors are attracting a great deal of attention for a wide range of industrial applications, ranging from oil\&gas to structural health monitoring, energy production and transportation [1]. They offer several advantages over conventional electronic sensors in terms of compactness, immunity to electromagnetic interference, resistance to harsh environments and multiplexing capabilities. Wavelength division multiplexed FBG sensors can be interrogated using low cost miniaturized solid state read-out units, making the sensing technology particularly attractive for high-volume market sectors and for specific applications requiring robust and reliable solutions for harsh environments. In this context, the silicon on insulator (SOI) platform is particularly suitable for the realization of high-density photonic integrated circuits (PIC) exploiting complementary metal-oxide-semiconductor (CMOS) compatible processes, which potentially allows the combination of both photonic and electronic functionalities into the same chip [2]. Although silicon lacks the capability of lasing and detecting light at the telecom wavelengths, many efforts have been focused on integrating active components on the same platform; while photodetectors can already be integrated in SOI either by hybrid integration of III-V components or by direct growth of germanium photodiodes, laser sources can be coupled off-chip, bonded and possibly directly grown in the future [3]. Comparing cost and performance provided by the different technology platforms, silicon-based devices seem to be the most attractive solution.

In [4], we demonstrated an integrated version of the scheme proposed in [5], which is based on an actively monitored unbalanced Mach-Zehnder interferometer (MZI) using the phase-generated carrier (PGC) demodulation technique, showing wavelength division multiplexing interrogation of two FBGs by using a single MZI and an external AWG. In this work we follow up by integrating both the MZI and the AWG on the same chip. Input and output grating couplers were used to vertically couple the signals reflected from the FBGs to the chip and the AWG output signals were coupled to external photodetectors. The integration of photodetectors and light source on a SOI platform has been already demonstrated and will be the subject of future developments.

\section{Theory}

A single unbalanced MZI demodulator can be used to convert multiple FBG sensors Bragg wavelength shifts into phase variations of the output signals at the photodetectors, allowing for very high resolution WDM interrogation. This type of MZI is characterized by a differential length $d$ between its arms and by a free spectral range (FSR) defined as the spectral period of the fringe pattern. If the spectral width of each FBG is narrower than half the FSR of the MZI and falls within the transmission bandwidth of one port of the AWG following the MZI, an interference signal will be obtained for each wavelength multiplexed FBG at the output of the AWG. Assuming for simplicity unitary and flat transmission of the AWG, this output signal can be written as $I=A+B \cos \Delta \varphi(t)$, where $A$ is the DC component, $B$ is proportional to the input intensity and depends on the mixing efficiency of the MZI, and $\Delta \varphi(t)$ is the phase difference between the signals in each arm of the MZI.

Active phase demodulation schemes like the phase generated carrier (PGC) technique allow a precise estimation of the phase variations of the signal of interest b6y applying an external phase modulation on one of the MZI arms, with a frequency much higher than that of the signal of interest [5]. Considering a sinusoidal modulating signal with frequency $\omega_{0}$ and amplitude $C=0.84 \pi$, the output of the MZI becomes $I=A+B \cos \left[C \cos \left(\omega_{0} t\right)+\Delta \varphi(t)\right]$, which can be expanded in terms of Bessel functions. By mixing each output of the AWG with the proper even and odd multiple of $\omega_{0}$, in this case $\omega_{0}$ and $2 \omega_{0}$, and by applying a low-pass filter to the signals to remove the frequencies above the band of interest, it is possible to obtain two signals, $S_{1}$ and $S_{2}$, that are proportional to $\sin \Delta \varphi(t)$ and $\cos \Delta \varphi(t)$ respectively for each $\mathrm{FBG}$, from which the phase variations can be extracted by using, for example, the arc-tangent demodulation technique as $\Delta \varphi(t)=\arctan \left(\mathrm{S}_{1} / \mathrm{S}_{2}\right)$; finally, this 
phase change can be converted into wavelength shift $\Delta \lambda$ relative to the MZI free spectral range $\Delta \lambda_{F S R}$, by using the relation $\Delta \lambda=\Delta \lambda_{\mathrm{FSR}} \Delta \varphi(\mathrm{t}) / 2 \pi$.

Note that the same MZI can be used to monitor many different FBGs simply by wavelength demultiplexing its output using the AWG; this means that only one modulator is needed to track the wavelength of many different gratings simultaneously.

\section{Design and fabrication}

The device (see Fig. 1.a) was fabricated from 8 "SOI wafers with $220 \mathrm{~nm}$ silicon thickness and $2 \mu \mathrm{m}$ buried oxide. Deep-UV 193nm optical lithography [6] was used to pattern 220nmx480nm single-mode waveguides. The light was coupled from a single mode fiber to focusing grating couplers at $11^{\circ}$, characterized by a coupling loss of $\sim 5 \mathrm{~dB}$. The photonic circuit included two MZIs, one with path difference $\Delta \mathrm{L}=118.6 \mu \mathrm{m}$ and FSR of $4.8 \mathrm{~nm}(600 \mathrm{GHz})$, so called coarse, and another with $\Delta \mathrm{L}=948.7 \mu \mathrm{m}$ and FSR of $595 \mathrm{pm}(75 \mathrm{GHz})$, so called fine. The short branch of each MZI included a thermal phase modulator, consisting of a metal Ti/TiN heater track with length of $86 \mu \mathrm{m}$ and width of $1.43 \mu \mathrm{m}$, which had a $\mathrm{V}_{2 \pi}$ of $3.21 \mathrm{~V}$, and a $1 / \mathrm{e}$ time constant of $7 \mu \mathrm{s}$. The output of the MZI was connected to an AWG which was generated using the filter toolbox from Luceda Photonics. It featured eight $600 \mathrm{GHz}$-spaced channels and a single-etch profile. The insertion loss was $4.3 \mathrm{~dB}$ and the channel isolation was $15.3 \mathrm{~dB}$. The measured spectral response of the AWG device and a comparison with the FBGs spectral width is reported in Fig. 1.b. The outputs of the AWG were connected to grating couplers and collected with a fiber array. The overall footprint of the system was $1430 \mu \mathrm{mx} 400 \mu \mathrm{m}$.

(a)

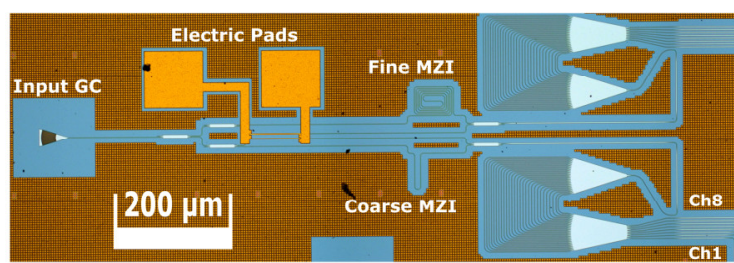

(b)

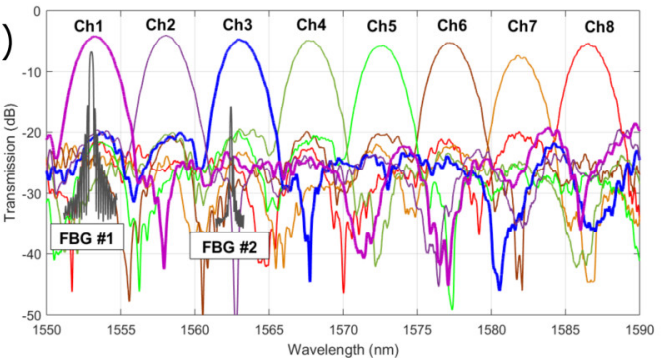

Figure 1. (a) Optical microscope image of the fabricated device with footprint of $1430 \mu \mathrm{mx} 400 \mu \mathrm{m}$. GC: grating coupler. (b) Measured spectral response of the device and comparison with the FBGs spectral width.

\section{Experimental results}

The experimental setup used for the dynamic characterization of the FBGs based on PGC demodulation is shown in Fig. 2; the output of a wideband light source from BaySpec Inc. (centered at $1550 \mathrm{~nm}$ with a spectral width of $100 \mathrm{~nm}$ ) is amplified using a high power EDFA, and then coupled to the FBGs under test using a threeport optical circulator (OC). For the strain measurements two FBGs were used. The FBG \#1, from Innovative Fibers Inc., characterized by a Bragg wavelength of $\sim 1552.78 \mathrm{~nm}$, a 3-dB bandwidth of $250 \mathrm{pm}$ and $\sim 85 \%$ reflectivity, was strained by the vibrations generated by a piezo speaker, whereas FBG \#2, from FBGS Technologies $\mathrm{GmbH}$ and characterized by a Bragg wavelength of $1560 \mathrm{~nm}$, a 3-dB bandwidth of $\sim 100 \mathrm{pm}$, and $\sim 15 \%$ reflectivity, was strained using a piezo-electric actuator (PZT). The FBGs were pre-strained in order to apply strain effectively and to place the FBGs close to the transmission maxima of the AWG, resulting in Bragg wavelengths of $\sim 1553.1 \mathrm{~nm}$ (FBG \#1) and $1562 \mathrm{~nm}$ (FBG\#2), as shown in Fig. 1.b. The reflection from the FBGs was divided using a $90 / 10$ coupler; the $10 \%$ of the signal was sent to a commercial readout unit from Bayspec Inc., while the $90 \%$ part was coupled to the device under test. The PIC output was then coupled to InGaAs based photoreceivers with trans-impedance amplifiers and $12 \mathrm{kHz}$ bandwidth. Finally, the photoreceiver electrical signals are connected to a DAQ $(100 \mathrm{kS} / \mathrm{s})$ for data acquisition and processing on a PC. 


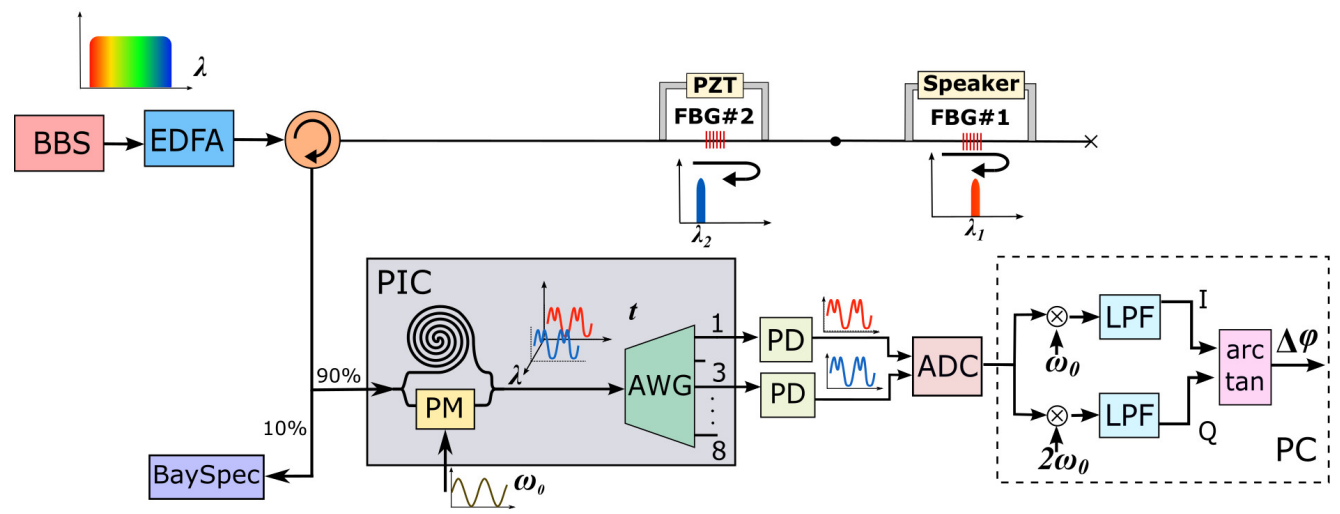

Figure 2. Experimental Setup. BBS: Broadband Source; EDFA: Erbium-doped fiber amplifier; PZT: Piezo-Electric Actuator; PIC: Photonic Integrated Circuit; PM: Phase Modulator; AWG: Array Waveguide Grating; PD: Photodetector; ADC: Analog-to-Digital Converter; LPF: Low-Pass Filter; PC: Personal Computer.

To demonstrate the capabilities for multiple FBG interrogation, a sinusoidal signal of $210 \mathrm{~Hz}$ of frequency and $500 \mathrm{mV}$ peak-to-peak amplitude was applied to the driver of the speaker straining FBG \#1, while a sinusoidal signal of $140 \mathrm{~Hz}$ and $2.7 \mathrm{~V}$ peak-to-peak with $1.35 \mathrm{~V}$ of DC component was applied to the PZT driver in order to strain FBG \#2. Simultaneously, the phase modulator in one of the arms of the MZI was driven using the square-root of a sinusoidal signal, since the applied power is proportional to the square of the voltage. The peak-to-peak amplitude was $2.92 \mathrm{~V}$, which satisfies the condition $\mathrm{C}=0.84 \pi$, and the frequency was $5.8 \mathrm{kHz}$, which is more than ten times the frequency of variation of the Bragg wavelengths.

Fig. 3.a compares the estimated wavelength shift for FBG \#1 obtained by applying the PGC demodulation technique while monitoring the coarse MZI and the commercial FBG readout unit from BaySpec Inc.. Note that the integrated device accurately measures variations of approximately 344.6 pm peak-to-peak, presenting a good agreement with the commercial readout unit results.
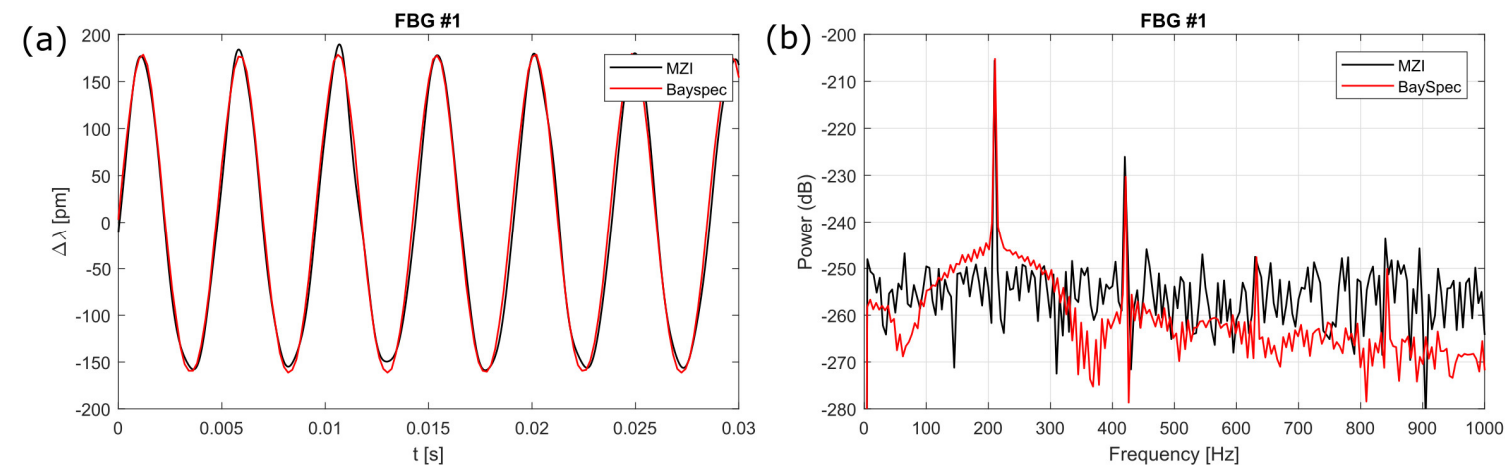

Figure 3. Comparison of (a) the measured wavelength shift versus time obtained with the device under test (in black) and the commercial FBG readout from BaySpec Inc. (in red) for FBG \#1, and (b) the spectra of the measured wavelength variations for each device.

The spectra of the measured wavelength shift signals for FBG \#1 are shown in Fig. 3.b. A signal-to-noise ratio (SNR) normalized to a $1-\mathrm{Hz}$ bandwidth of approximately $48.18 \mathrm{~dB}$ was measured for the PIC, whereas for the BaySpec was approximately $64.1 \mathrm{~dB}$. The performance of the Bayspec is slightly better than the one of the proposed integrated solution, which could be due to the low signal intensity at the output of the device with respect to the noise of the photoreceiver. The PIC performance could be improved by reducing the total circuit loss by integrating the photoreceivers in the same chip.

Fig. 4.a shows the comparison of the estimated wavelength shift for FBG \#2 obtained with the proposed circuit and the commercial FBG readout unit from BaySpec Inc.. In this case the measured wavelength shift obtained with the BaySpec was 104 pm peak-to-peak but the result obtained with the integrated circuit presents some distortion and the amplitude does not have such a good agreement as the one obtained for FBG \#1, since FBG\#2 had a lower reflectivity, which translates into a lower SNR and more susceptibility to the crosstalk effect of the AWG. 
(a)

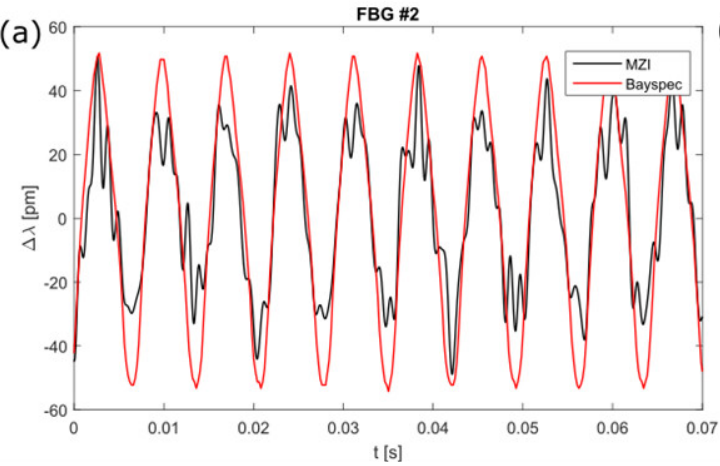

(b)

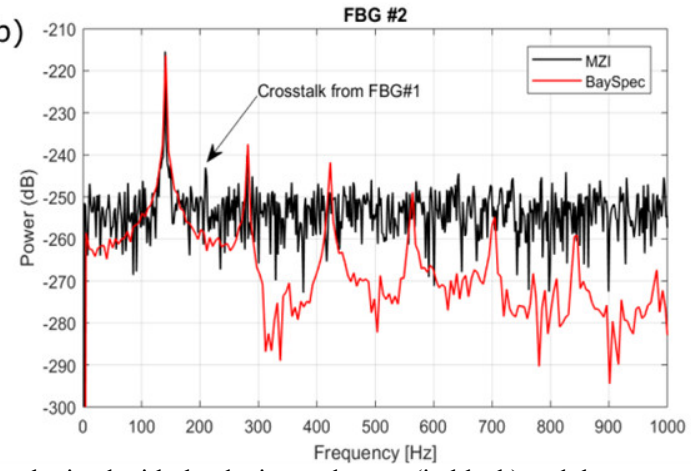

Figure 4. Comparison of (a) the measured wavelength shift versus time obtained with the device under test (in black) and the commercial

FBG readout from BaySpec Inc. (in red) for FBG \#2, and (b) the spectra of the measured wavelength variations for each device.

The spectra of the measured wavelength shift signals for FBG \#2 are shown in Fig. 4.b. A signal-to-noise ratio (SNR) normalized to a 1-Hz bandwidth of approximately $38.8 \mathrm{~dB}$ was measured for the PIC, whereas for the BaySpec was approximately $56.8 \mathrm{~dB}$. The lower performance for FBG \#2 could be explained by the lower power reflected by the FBG and by the presence of a harmonic corresponding to the frequency of the straining signal of FBG \#1 at $210 \mathrm{~Hz}$, as shown in Fig. 4.b. The performance of the device could be improved by employing a higher reflectivity FBG and by integrating the photoreceivers in the same chip.

\section{Conclusion}

We presented an integrated FBG interrogator based on an actively monitored unbalanced MZI using the PGC technique. The device was fabricated on a SOI platform and it is capable of demodulating the wavelength shift variations of multiple FBGs simultaneously, by using an integrated AWG at the output of the MZI. The accuracy of the measurements was validated by comparing with a commercial readout unit, obtaining a good agreement in the case of the FBG with high reflectivity and a lower performance for the FBG with lower reflectivity. The use of high reflectivity FBGs and the integration of the photoreceivers in the same SOI platform could improve the overall performance and will be the subject of future work.

\section{Acknowledgment}

This work is partially supported by Regione Toscana PAR FAS 2007-2013 grant STech, Regione Toscana POR FESR 2014-2020 grant MIIID, and EU FP7 project IRIS (contract 619194).

\section{References}

[1] B. Culshaw and A. Kersey, "Fiber-Optic Sensing: A Historical Perspective," Journal of Lightwave Technology, pp. 1064-1078 (2008).

[2] L. C. Kimerling et al., "Electronic-photonic integrated circuits on the CMOS platform," Silicon Photonics , Proc. of SPIE, vol. 6125 612502 (2006).

[3] K. Tanabe, K. Watanabe, Y. Arakawa, "III-V/Si hybrid photonic devices by direct fusion bonding", Scientific Reports, Vol. 2, Article number: 349 (2012).

[4] Y. Marin, et al., "Integrated FBG Sensors Interrogation using Active Phase Demodulation on a Silicon Photonic Platform," Journal of Lightwave Technologies, vol. 35, no. 6, pp. 3374-3379 (2017).

[5] A. Dandridge, A. B. Tveten, and T. G. Giallorenzi, "Homodyne demodulation scheme for fiber optic sensors using phase generated carrier," IEEE Trans. Microw. Theory Techn., vol. 30, no. 10, pp. 1635-1641 (Oct. 1982).

[6] C. Kopp et al., "Silicon photonic circuits: On CMOS integration, fiber optical coupling and packaging," IEEE J. Sel. Topics Quantum Electron., vol. 17, no. 3, pp. 498-509 (May/Jun. 2011). 